Reprod. Nutr. Dévelop., 1980, 20 (3 B), 897-903.

\title{
Modèle théorique du développement de la sensibilité aux stimulations spécifiques intervenant dans les relations parentales
}

par P. JAISSON

\author{
Laboratoire d'Ethologie et Sociobiologie, \\ Equipe de Recherche Associée au CNRS no 885 \\ Université Paris XIII, \\ avenue J.-B.-Clément, 93430 Villetaneuse, France.
}

\begin{abstract}
Summary. A theorefical model of the development of specific stimulation sensitivity in parental relationships.

A comparison of six studies on the development of specific stimulation recognition in parental relationships reveals some common points. In all cases, the recognition of complex specific stimulations is obtained by practice (in contact with the environment) in dealing with a simple stimulation which does not seem to be learned. The configuration of the final complex specific stimulation is associated with the simple, initial stimulation, both of them belonging to the same sensory register. The efficiency of the new stimulation makes the first one obsolete.

From these convergences we have constructed a working model to determine the profile of individual sensitivity in time : the simple, initial stimulation would complement a rigid sensitivity site, situated within a flexible, pliable sensitivity, which would be modeled by and rigidified at the contact of the complex stimulations associated with the efficient simple stimulation. In the initiation of a same behavior, a time relation is thus established between the basic, simple specific stimulation and the final complex stimulation.

The new rigid sensitivity of the individual could eventually serve as a starting point for another sensitivity, corresponding to a still more complex stimulation.
\end{abstract}

L'avancement actuel des recherches en éthologie, en particulier les travaux sur l'éthogenèse (ou développement du comportement), permet d'engager des réflexions sur un modèle heuristique du développement de la sensibilité aux stimulations spécifiques dans les relations parentales. En effet, un certain nombre d'analyses du développement des relations parentales chez l'animal et chez l'homme rendent possible, à mon sens, une telle tentative, même prudente. S'il était réalisable, un modèle conceptuel aurait la seule prétention de mettre un peu d'ordre dans nos connaissances et de faciliter l'établissement d'hypothèses de recherche dans les travaux futurs.

Contrairement à beaucoup de recherches théoriques qui, souvent, consistent à manier les idées avant de s'intéresser aux faits, notre démarche essaiera d'aboutir à un certain niveau d'abstraction à partir de la base concrète de l'expérimentation. Après avoir brièvement résumé les résultats de six recherches distinctes portant 
sur l'établissement des relations parentales dans divers groupes zoologiques, nous examinerons leurs points communs, voire leur éventuelle unicité fondamentale. L'hypothèse de base, commune à tous ces travaux, est l'absence d'une reconnaissance spontanée de certaines stimulations spécifiques complexes (qui correspondraient aux anciens « déclencheurs » des éthologistes objectivistes) mais au contraire une élaboration précoce de cette reconnaissance par une éthogenèse intégrant des informations indispensables du milieu.

Les différents exemples retenus sont :

- la reconnaissance du parent impliquée dans le comportement de poursuiłe précoce (ou « empreinte de poursuite ») chez les poussins d'oiseaux nidifuges, par exemple l'Oie cendrée (Lorenz, 1935) ;

- la reconnaissance de la tête du parent impliquée dans le comportement de sollicitation alimentaire chez le poussin de Mouette rieuse (Hailman, 1967) ;

- la reconnaissance de la tétine maternelle chez le Chaton (Rosenblatt, 1972) ;

- la reconnaissance du visage de la mère, provoquant le sourire chez l'enfant humain (Kagan et Lewis, 1965 ; Kagan, 1970) ;

- la reconnaissance du cri maternel chez le Caneton Colvert (Gottlieb, 1976) ;

- ia reconnaissance de la nymphe chez la jeune Fourmi rousse (Jaisson, 1975).

Les six exemples sont comparés, pour ce qu'ils ont de commun, dans le tableau synoptique.

Tableau synoptique comparatif pour les six exemples de développement de la reconnaissance des stimulations intervenant dans les relations parentales

\begin{tabular}{|c|c|c|c|c|c|}
\hline Espèce & Auteur & $\begin{array}{l}\text { Comportement } \\
\text { impliqué }\end{array}$ & $\begin{array}{l}\text { 1er Stimulus } \\
\text { efficace }\end{array}$ & $\begin{array}{c}2 \text { e Stimulus } \\
\text { efficace }\end{array}$ & $\begin{array}{l}\text { Motivation et/ou } \\
\text { stimulations proba- } \\
\text { blement associées }\end{array}$ \\
\hline $\begin{array}{l}\text { Oje Cendrée } \\
\text { (poussin) }\end{array}$ & $\begin{array}{c}\text { Lorenz } \\
(1935)\end{array}$ & $\begin{array}{l}\text { Poursuite de la } \\
\text { mère }\end{array}$ & Objet mobile & Mère & $\begin{array}{l}\text { Renforcement alimen. } \\
\text { taire-fraterie-cris ma. } \\
\text { ternels, ... etc. }\end{array}$ \\
\hline $\begin{array}{c}\text { Mouette rieuse } \\
\text { (poussin) }\end{array}$ & $\begin{array}{l}\text { Hailman } \\
(1967)\end{array}$ & $\begin{array}{l}\text { Sollicitation ali- } \\
\text { mentaire des } \\
\text { parents }\end{array}$ & Bec rouge & Tête de l'adulte & $\begin{array}{l}\text { Renforcement alimen. } \\
\text { taire, mobilité du Sti. } \\
\text { mulus }\end{array}$ \\
\hline $\begin{array}{c}\text { Chat } \\
\text { (nouveau-né) }\end{array}$ & $\begin{array}{l}\text { Rosenblatt } \\
(1972)\end{array}$ & $\begin{array}{l}\text { Choix de la téti- } \\
\text { ne maternelle }\end{array}$ & $\begin{array}{l}\text { Eléments tactiles } \\
\text { non spécifiques } \\
\text { (périphérie des } \\
\text { tétines) }\end{array}$ & $\begin{array}{l}\text { Configuration tac- } \\
\text { tile spécifique de } \\
\text { la tétine connue }\end{array}$ & $\begin{array}{l}\text { Renforcement alimen. } \\
\text { taire, Chaleur corpo- } \\
\text { relle, odeur du lait,... } \\
\text { etc. }\end{array}$ \\
\hline $\begin{array}{c}\text { Homme } \\
\text { (nourrisson) }\end{array}$ & $\begin{array}{l}\text { Kagan } \\
(1970)\end{array}$ & $\begin{array}{l}\text { Sourire du } \\
\text { nourrisson }\end{array}$ & $\begin{array}{l}\text { Silhovette non né- } \\
\text { cessairement fi- } \\
\text { gurative du visa- } \\
\text { ge d'un adulte }\end{array}$ & $\begin{array}{l}\text { Silhouette figurati- } \\
\text { ve du visage d'un } \\
\text { adulte, puis de la } \\
\text { mère }\end{array}$ & $\begin{array}{l}\text { Renforcement alimen- } \\
\text { taire et social }\end{array}$ \\
\hline $\begin{array}{c}\text { Canard Colvert } \\
\text { (poussin) }\end{array}$ & $\begin{array}{c}\text { Gotflieb } \\
(1976)\end{array}$ & $\begin{array}{l}\text { Reconnaissance } \\
\text { du cri mater- } \\
\text { nel }\end{array}$ & $\begin{array}{c}\text { Fréquences basses } \\
\text { du cri maternel }\end{array}$ & $\begin{array}{l}\text { Cri maternel com- } \\
\text { plet }\end{array}$ & $?$ \\
\hline $\begin{array}{l}\text { Fourmi rousse } \\
\text { (jeune imago) }\end{array}$ & $\begin{array}{c}\text { Jaisson } \\
(1975)\end{array}$ & $\begin{array}{l}\text { Reconnaissance } \\
\text { des pupes par } \\
\text { la jeune ou- } \\
\text { vrière }\end{array}$ & $\begin{array}{l}\text { Odeur supra-gé- } \\
\text { nérique }\end{array}$ & Odeur de l'espèce & Renforcements sociaux \\
\hline
\end{tabular}


1. - La réaction de poursuife fut probablement l'exemple le plus classique dans l'étude du phénomène d'imprégnation. Lorenz en a réalisé l'analyse chez plusieurs oiseaux nidifuges, en particulier l'Oie cendrée (1935) : peu de temps après la sortie de l'œuf, le poussin commence à poursuivre spontanément un objeł mobile présent dans son environnement proche. La configuration de cet objet est peu importante, à la condition que son volume soit compris entre un minimum et un maximum, au-delà duquel la réaction provoquée tendrait plutôt vers une fuite. Dans les conditions naturelles il y a évidemment peu de chances pour que cet objet mobile soit autre chose que la propre mère du poussin. Mais en conditions expérimentales, ce dernier peut aussi bien poursuivre un objef inerte, un autre animal ou un être humain. L'établissement d'une « relation objectale » avec la mère (naturelle ou artificielle) semble bien lié à l'apparition de l'évitement face à tout objet mobile inconnu. Cette nouvelle réaction se substitue donc à l'attraction spontanée manifestée plus précocement. En conséquence, le même comportement de poursuite spontanée n'apparaît plus pour toute une famille d'objets mobiles possibles, mais pour le seul objet maternel familier. L'information portée par ce dernier est complexe : c'est un stimulus beaucoup plus fin que celui auquel l'animal était potentiellement capable de répondre à la naissance. Le même objet mobile n'a donc plus, pour le poussin, la même signification. Toutefois, le nouveau stimulus efficace reste visuel, même si le jeune animal à intégré, par d'autres voies sensorielles des informations (auditives par exemple) qui complètent cette reconnaissance.

2. - Quelques heures après l'éclosion le poussin de la Mouette rieuse manifeste ses premiers comportements de sollicitation alimentaire, consistant à agripper avec son bec celui de l'adulte (père ou mère) qui répond par une régurgitation réflexe. Ce comportement est donc indispensable à la survie du jeune. Hailman (1967) a démontré qu'à cet âge le poussin peut manifester une sollicitation alimentaire à la seule présence du bec de l'adulte, uniformément rouge chez la mère comme chez le père ; mais, après avoir vécu une semaine dans le nid familial, le jeune ne réagit plus au bec rouge; il répond uniquement à une représentation d'une tête complète de Mouette (bec rouge + tête noire). Là encore le même comportement n'apparaît qu'en présence d'un stimulus nouveau, plus complexe que le premier qu'il inclut pourtant (simple bec rouge). Hailman semble avoir démontré l'importance de la motivation alimentaire dans ce transfert : un conditionnement opérant interviendrait. Comme dans l'exemple précédant le registre sensoriel impliqué reste le même : un stimulus visuel est remplacé par un autre stimulus visuel auquel l'animal répond significativement.

3. - Lors de sa première recherche de la tétine maternelle le tout jeune Chaton est d'abord guidé par la chaleur et par l'odeur que porte la fourrure ventrale de la mère puis par des stimulations tactiles liées à l'ornementation de la peau entourant chaque tétine (Rosenblatt, 1972). Lors des prises alimentaires ultérieures le Chaton manifestera une préférence pour la tétine qu'il a saisie la première fois par hasard. En fait, le dessin formé par la peau autour de chaque tétine est particulier et engendre des stimulations tactiles de la bouche du Chaton, différentes de celles que peut provoquer la périphérie d'une autre tétine. II semble que cette fidélité à la première tétine soit due à la reconnaissance spécifique de l'ornementation de la peau qui l'entoure. 
lci, le même comportement de succion répond, au départ, à la présence de stimulations tactiles communes aux tétines puis à des stimulations propres à celle rencontrée pour la première fois. Dans cet affinement de la perception du stimulus tactile efficace intervient, en association, la sensibilité à la chaleur du corps de la mère ainsi qu'à l'odeur du lait.

4. - Le sourire de l'enfant humain semble apparaître spontanément à l'âge de quelques semaines, en réponse à la présence simultanée des éléments du visage de l'adulte (nez, bouche, yeux ef sourcils). Il apparaît même lorsque ces éléments sont disséminés sur un leurre dans une configuration aberrante (fig. 1) sans différence

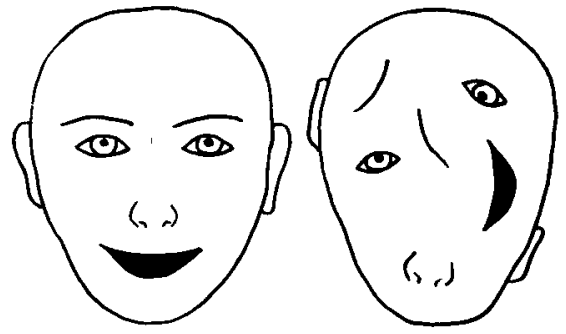

FIG. 1. - Représentation des leurres de visage utilisés pour le déclenchement du sourire chez le nourrisson, d'après Kagan, (1970).

significative avec un leurre conforme à l'organisation normale du visage (Kagan, 1970). Toutefois, si l'on retire trop d'éléments à ce schéma présenté au nourrisson, en ne laissant qu'un œil par exemple, aucune réaction n'est déclenchée. De trois mois à la fin du sixième mois environ le même enfant ne manifestera son sourire que face au seul leurre figuratif. Il devient donc « exigeant » quant à l'organisation de la stimulation significative. Plus tard, vers sept mois, il n'accordera plus son sourire spontané qu'au seul visage de sa mère ou la (ou les) personne(s) s'occupant régulièrement de lui. Dans cet exemple choisi chez notre espèce la stimulation efficace pour l'apparition d'un même comportement évolue encore dans le sens d'un affinement perceptif situé dans le même registre sensoriel (visuel).

5. - Gottlieb (1976) a recherché pourquoi le Caneton Colvert à l'éclosion reconnaît déjà le cri maternel de son espèce. Ceci est le résultat d'une éthogenèse dans laquelle interagissent maturation et expérience. En effet si l'on rend muet un embryon en engluant ses cordes vocales ef si on l'isole seul dans un incubateur (afin de le soustraire aux émissions vocales d'autres embryons), cet animal isolé-dévocalisé sera incapable de différencier, à l'éclosion, le cri maternel de son espèce de celui de la poule. En l'absence d'une expérience auditive prénatale le Caneton n'est sensible qu'aux fréquences basses, communes au cri maternel de la Cane Colvert et à celui de la poule qui s'y trouve entièrement localisé. Seul l'animal ayant subi l'expérience prénatale de ses propres cris ou de ceux de ses frères de couvée pourra être sensible aux fréquences moyennes existant uniquement dans le cri maternel de la Cane Colvert. C'est pourquoi un Caneton normal préférera se diriger vers un haut-parleur produisant le cri maternel d'une Cane Colvert plutôt que vers celui qui, simultanément, émef le cri maternel d'une poule. Ce comportement normal existe également chez le Caneton isolé-dévocalisé au stade embryonnaire mais qui a subi l'audition forcée des cris dits de «contact-contentement », enregistrés chez un autre embryon. Dans cet exemple, ce sont des signaux auditifs dont la signification est précisée au 
cours de l'éthogenèse. Comme le souligne Gottlieb, il est a priori difficile d'imaginer l'association de motivations à ce développement comportemental car le cri de « contactcontentement» est efficace alors que l'embryon isolé-dévocalisé devrait être plus logiquement motivé pour émettre un cri dit de « détresse ». Cependant, localisé dans les hautes fréquences, ce dernier est inefficace pour le développement de la reconnaissance des fréquences moyennes du cri maternel. L'expérience prénatale est donc indispensable au Caneton; mais notons qu'elle est obligatoire dans les conditions naturelles où l'animal n'a pas la possibilité d'apprendre autre chose que ses propres cris : ses conditions de vie et ses propriétés fonctionnelles exercent une contrainte sur le type d'apprentissage pouvant être réalisé.

6. - La jeune Fourmi rousse venant d'éclore ne préfère pas spontanément les pupes de son espèce (Jaisson, 1975). A ce stade, l'ouvrière est en fait capable de manifester des comportements de soins à l'égard de pupes hétérospécifiques, pouvant même appartenir à un genre différent. Cependant elle ne réagit pas à la présence de pupes d'un genre très éloigné phylogénétiquement. Lorsque, immédiatement après la naissance, la jeune Fourmi est placée au contact de pupes d'un genre voisin pendant une à deux semaines elle refusera ensuite de soigner celles de sa propre espèce, qui seront simplement dévorées. Le type de pupe connu lors de la période post-natale sera seul l'objet de soins. Dans les conditions naturelles le contraire est évidemment observé : seules les pupes homospécifiques sont soignées par les Fourmis, tout cocon étranger étant détruit. Il a été démontré que cette reconnaissance est basée sur la discrimination de substances actives (ou phéromones) dont la nature chimique est encore inconnue. Dans cet exemple, la sensibilité de l'animal aux stimulations efficaces évolve encore en fonction de son expérience précoce : immédiatement après la naissance il est sensible à une stimulation chimique non spécifique de l'espèce mais commune, au contraire, à tout un groupe de genres ; puis, au terme d'une expérience précoce, seules les pupes portant l'odeur spécifique apprise pourront recevoir des soins. Un affinement perceptif est de nouveau caractérisé dans le cadre d'un même registre sensoriel, cette fois-ci olfactif.

Les figures 2 à 4 tendent à schématiser un modèle théorique qui peut être élaboré à travers la comparaison des différents exemples rapidement résumés ci-dessus.

La figure 2 met en valeur la faible exigence de la sensibilité spontanée de l'animal à un premier stimulus quant au niveau de complexité de ce dernier : dans une sensibilité «souple » de l'individu (représentée par la surface ondulée) s'insèrent en empreinte des sites de sensibilité « rigide ». Dans un état de motivation favorable, pour déclencher un comportement correspondant les stimulations externes doivent s'adapter à ces sites de sensibilité « rigide » de façon stéréospécifique. Au cours de l'éthogenèse, différentes stimulations potentielles du milieu peuvent assurer cette complémentarité, à la condition de posséder entre elles une région commune pouvant s'ajuster à un site de sensibilité spontanée. Au cours de l'étape suivante, exprimée dans la figure 3, la sensibilité « rigide» de l'individu se complique au contact du stimulus par «durcissement » de la sensibilité « souple » contiguë. Cette différenciation d'une nouvelle sensibilité « rigide» plus précise, plus fine, réduira le nombre des stimulations efficaces pouvant engendrer le même comportement. Au terme de ce développement un relai s'est trouvé établi entre une sensibilité spontanée relative- 


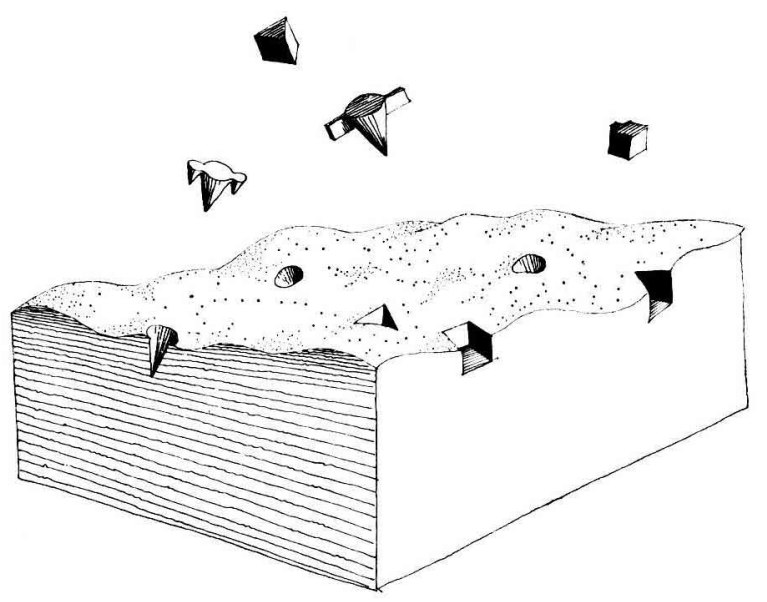

FIG. 2.

FIG. 3 .
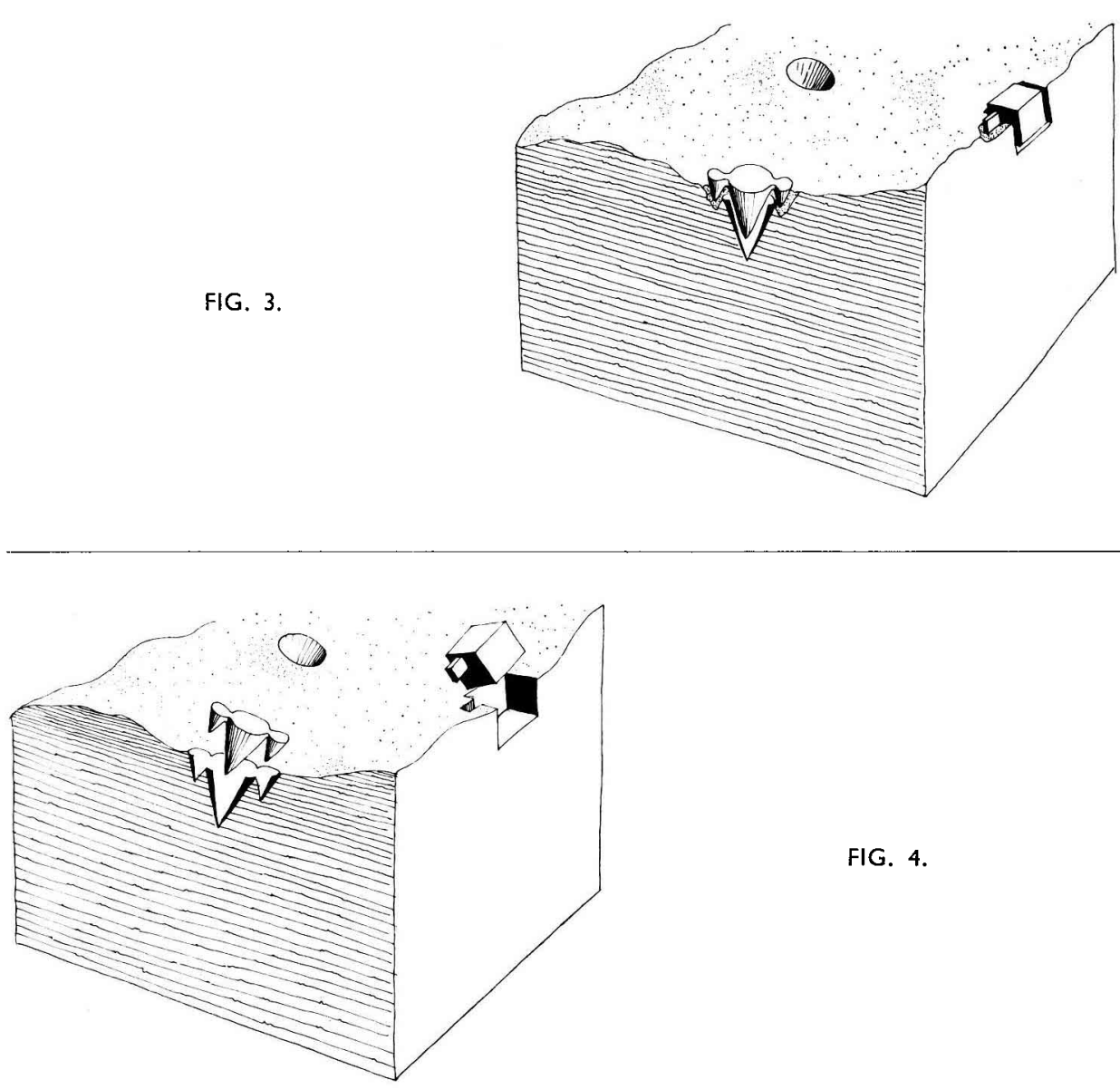

FIG. 4. 
ment fruste et une sensibilité élaborée à travers l'expérience de l'environnement précoce. La sensibilité « rigide » du début est un élément obligatoire, une contrainte sur le développement d'une sensibilité nouvelle, tandis que l'activité comportementale impliquée reste apparemment la même. Cette évolution se réalise dans le même registre sensoriel, mais des stimulations d'une sensorialité étrangère peuvent aider. S'agissant d'un phénomène précoce dans la vie de l'individu, son importance sera considérable dans le registre des relations parentales. La nouvelle sensibilité « rigide » obtenue pourra être une simple transition vers l'acquisition d'une autre sensibilité encore plus fine, et ainsi de suite... Toutefois, il est possible que la propriété de compliquer la structure des sites de sensibilité spécifique par « durcissement » n'existe qu'à certains moments de la vie de l'individu, ce qui correspondrait à ce que l'on appelle par ailleurs des périodes sensibles. Enfin, ce modèle théorique, est compatible avec la possibilité de réversibilité par des différenciations partielles des sites jusqu'au stade initial, suivi d'une nouvelle période sensible permettant la reconnaissance d'une nouvelle stimulation : ce pourrait être le cas de l'« empreinte parentale » répétée à chaque parturition, découverte par exemple par Klopfer et al. (1964) chez la Chèvre.

Ce modèle heuristique, certes très imparfait, n'est qu'une première esquisse que je livre à la sagacité des critiques (que j'espère constructives) dans le but d'aboutir à une meilleure élaboration théorique pouvant aider la réalisation de recherches futures. Les résultats expérimentaux de l'Ethologie contemporaine deviennent suffisamment nombreux pour pouvoir investir dans une direction théorique. Il est à souhaiter qu'un dialogue s'établisse entre physiologistes de la perception ef éthologistes afin de rechercher un éventuel modèle synthétique qui aurait en outre l'avantage de matérialiser la notion quelque peu confuse de «filtre » souvent utilisée pour expliquer les sensibilités (ou les réactivités) différentielles.

Colloque «La relation parentale » Nouzilly, France, juin 1979.

\section{Références}

GOTTLIEB G., 1976. Early development of species-specific auditory perception in birds, 235-280. In GOTTLIEB G., Neural and behavioral specificity. Studies on the development of behavior and the nervous system, 3, Acad. Press, New York.

HAILMAN J. P., 1967. The ontogeny of an instinct : the pecking response in chicks of the laughing Gull (Larus atricilla L.) and related species. Behaviour, Suppl. XV, 159 pp.

JAISSON P., 1975. Contribution à l'éfude de l'expérience précoce chez les Fourmis. Th. Doct. Etat, Univ. P. et M. Curie, Paris, 175 pp.

KAGAN J., 1970. Attention and psychological change in the child. Science, 170, 826-832.

KAGAN J., LEWIS M., 1965. Studies of attention in the human infant. Merrill-Palmer Q., 11, 95-122.

KLOPFER P. H., ADAMS D. K., KLOPFER M. S., 1964. Maternal « imprinting 》 in goats. Proc. nat. Acad. Sci. U.S.A., 52, 911-914.

LORENZ K., 1935. Der Kumpan in der Umvelt des Vogels. J. Ornithol., 83, 137-312.

ROSENBLATT J. S., 1972. Learning in newborn Kittens. Sci. Amer., 227, 18-25. 